\title{
Assisted Reproduction Techniques and Acupuncture: Does it works? Literature review
}

\begin{abstract}
Objective: to review the role of acupuncture as a coadjutant therapy in assisted reproduction techniques (ART). Material and method: we used the search engines: Medline, Pubmed, Pubmed Central and Google Scholar; with the following keywords: "acupuncture in vitro fertilization", "acupuncture ivf", "acupuncture infertility", "traditional Chinese medicine ivf", "complementary medicine ivf".
\end{abstract}

Results: we found 189 articles and only 45 were selected. There were also 22 reviews and 7 metaanalysis from which we included 15 and 4 respectively.

Conclusion: The role of acupuncture as a coadjutant therapy in ART remains controversial. Future research should focus into following international guidelines in order to obtain clear and consistent results and reproducibility.

Keywords: acupuncture IVF, acupuncture infertility, traditional Chinese medicine IVF, complementary medicine IVF
Volume 9 Issue $6-2018$

\author{
Israel-Obed Carmona-Ruiz,' Pau Forcada \\ Caballé España, ${ }^{2}$ \\ 'Programme Director of the Master in Infertility at European \\ Foundation of Traditional Chinese Medicine San Antonio de \\ Murcia Catholic University, México \\ ${ }^{2}$ CEO European Foundation of Traditional Chinese Medicine, \\ Universitat Rovira i Virgili, Spain
}

\begin{abstract}
Correspondence: Israel-Obed Carmona-Ruiz, Programme Director of the Master in Infertility at European Foundation of Traditional Chinese Medicine, San Antonio de Murcia Catholic University, México, Institut Marques, Abbey Hall, Abbeylands, Clane, Co. Kildare.W9I N500, Ireland, Tel +353 45989 500, Email israel.carmona@institutmarques.ie
\end{abstract}

Received: December 19, 2017 | Published: December 31, 2018

\section{Introduction}

It is said that, traditionally, infertility affects between 8 and $12 \%$ of couples of reproductive age around the world. ${ }^{1,2}$ This varies in some regions where it can affect up to $30 \%$ of the population ${ }^{2,3}$ The regions with the highest prevalence include South Asia, North Africa, Central and Eastern Europe, among others. ${ }^{4}$ More recent estimates assert that one in four couples experience infertility. ${ }^{5}$

There are many causes of infertility: from ovulatory, peritoneal, and cervical problems, to male and genetic causes, among others. ${ }^{6}$ In addition, in almost half of couples with infertility, two or more factors coexist. $^{6}$

Infertility is recognized as a potentially serious, costly problem that not only has implications for health, but is a condition that is also linked to human rights. ${ }^{6-8}$ The social stigma that comes with it leads to isolation and neglect in many developed countries. ${ }^{9}$

Treating infertility should be aimed at the etiological causes, which will guarantee the highest chances of a successful pregnancy culminating in the birth of a healthy child. ${ }^{10}$ Basically, the three current therapeutic strategies may be summarized as:

\section{A. Pharmacological treatment \\ B. Surgical treatment \\ C. Assisted reproduction techniques $(\mathrm{ART})^{10}$}

In many cases, ART have become the first management option, primarily because they are less invasive than surgical treatment and offer a more successful outcome. However, globally, the success rates may still be considered low. In Europe, the success rate (clinical pregnancy rate) is less than $30 \%$ by ovarian drilling. ${ }^{11}$ Throughout the last decade, a large part of the research has been devoted to trying to increase the ART results, either by improving the quality of embryos, modulating hormones or controlling the environment within the uterus. $^{12}$
Complementary medicine emerges as a strategy of minimal invasion, due to its low cost, and it generally has no complications; therefore, this is preferred by women from the time when they are trying to get pregnant, to getting pregnant, during pregnancy and during menopause. ${ }^{13-15}$

Perhaps acupuncture is the most recognized technique when talking about complementary medicine. It is an important part of Traditional Chinese Medicine (TCM) and dates back at least 3,000 years. ${ }^{12}$ Its use in the West has become popular in the last decade, in particular in managing subfertility. However, its effectiveness remains controversial. ${ }^{16}$

Some studies have shown that acupuncture improves the results of ART. ${ }^{12,17}$ However, systematic reviews show contradictory results. ${ }^{16,18-21}$ One problem that systematic reviews and meta-analysis show is the heterogeneity of the management protocols as well as the treatment times. This causes the vast majority of studies to be discarded and so no conclusive statements can be drawn.

The present article is a review of the literature and a discussion about the deficiencies and strategies that clinical trials should follow regarding the use of acupuncture as a coadjuvant treatment for assisted reproduction procedures.

Objective: To carry out an analysis on the effects of acupuncture as a coadjuvant treatment in assisted reproduction procedures.

\section{Material and method}

A bibliographic search was made in the databases: Medline, PubMed, PubMed Central y Google Scholar. The following keywords were used: "Acupuncture in vitro fertilization", "acupuncture ivf", "acupuncture infertility", "traditional Chinese medicine ivf", "complementary medicine ivf". No date was selected as a filter, although searching was restricted to the English and Spanish languages. In addition, an article selection methodology was used based on the consistency, methodology, and the impact and clarity in 
the published results. With these premises, clinical trials, reviews and meta-analysis were chosen.

\section{Results}

The keywords that addressed the issue more generally (for example: "Acupuncture infertility") showed a maximum of 189 results, of which 45 articles were selected based on the criteria described above. In addition, 22 reviews and 7 meta-analyses were found, of which 15 and 4 studies were chosen, respectively.

\section{Discussion}

\section{Problematic in ART: its impact on health cost and results}

Regardless of the variability that exists when estimating its prevalence, it is known that infertility can be an important psychological factor in many couples who suffer from it..$^{22}$ Anxiety and depression are the most frequent signs related to diagnosis, treatment modalities, outcome and financial concerns. ${ }^{23}$ For example, women with recurrent implantation failures after IVF treatments suffer from significant stress and disappointment. ${ }^{24}$ Even if the treatment is successful, medical complications include multiple pregnancy or ovarian hyper-stimulation syndrome, which are conditions that can threaten patient's life. ${ }^{22}$

In vitro fertilization (IVF) was initially designed to treat the tubal factor. ${ }^{1,25}$ However, not only has its use been widened, there has also been significant globalization of ART due to the establishment of new clinics and reproduction centres in several countries. ${ }^{1}$ An example of this is that in the year 2000, ART was only available in 45 of the 191 (24\%) member countries of the World Health Organization (WHO). Ten years later, more than half of the nations offered ART services (105 countries, 55\% $)^{26}$ estimating the presence of between 4000 and 4500 ART clinics around the world. This number has grown to more than 5300 clinics in $2016^{5}$ however, these are not exact estimates due to the lack of participation of different countries and assisted reproduction centres in national and international registries.

Despite this "explosion" or globalization of ART, the cost per procedure is still high. It is known that, generally speaking, the private sector deals with most cases or cycles of patients due to different causes, among which is the fact that the public health sector is unable to finance treatments for all patients. This leads to a lack of regulation or reliable statistics about the real cost to patients. However, Papaleo et al. ${ }^{27}$ recently compared two embryo transfer strategies aiming to analyse the real costs of each of them in order to choose the best option. From this study it can be observed that the average to achieve a live birth varies between $€ 13,101$ and $€ 15,279$ according to the transfer policy used. ${ }^{27}$

These numbers show that in spite of the numerous advances in ART, the implantation and pregnancy rates do not increase significantly, and the cost-results ratio is still disproportionate. Dr. Robert Edwards estimated that $85 \%$ of embryos transferred in the uterine cavity fail to implant. ${ }^{28}$ To justify these low success rates, poor endometrial receptivity, poor embryo implantation capacity or suboptimal transfer techniques have been blamed.

In Europe, pregnancy rates by ovarian drilling and embryo transfer for IVF cycles are $29.4 \%$ and $33.8 \%$, respectively. ${ }^{11}$ In Spain, the
National Registry of the Spanish Fertility Society (SEF) publishes rates of $28.8 \%$ and $36.2 \%$ by puncture and transfer, respectively. ${ }^{29}$

If we relate the pregnancy rates, the cost and the prevalence of infertility, we can conclude that there is a need to improve these results by using techniques or strategies that lower the cost, improve the chances of becoming pregnant while at the same time being pleasant for patients.

\section{Acupuncture}

Acupuncture has become one of the most used treatments in alternative medicine. Its use in the West started more than 25 years ago and there are relatively few studies about it. Acupuncture has been used for the treatment of gynaecological disorders for several years. ${ }^{30}$ Many women consider it as an alternative therapy or coadjuvant treatment in the management of infertility. ${ }^{31}$

The explanation of the effects of acupuncture on the body is related to a type of sensory stimulation due to the insertion of needles into the skin and muscle. ${ }^{32}$ This stimulation can also be improved or modulated with an extra effect thanks to manual modifications or electrical stimulation with high and low frequencies. ${ }^{33}$ Moreover, acupuncture can have psychological effects that may be beneficial for some patients. ${ }^{34}$

The primary mechanism for the clinical effects of acupuncture from a western point of view is the somatic activation of the innervation of the skin and muscles, which in turn modulates nervous system activity and endocrine and metabolic functions. ${ }^{32}$

\section{Acupuncture: mechanism of action}

It is said that manual insertion or electrical stimulation of the needles placed on the skin and muscle, positively stimulates fibres $\alpha, \beta, \delta$ and $\mathrm{C}^{35}$ modulating the activity of the autonomic nervous system. ${ }^{36}$ Manual and electrical stimulation produces the release of neuropeptides from the peripheral nerve endings of the area surrounding the needle, increasing blood flow ${ }^{37}$ and glucose uptake by the skeletal muscle. ${ }^{38}$

For example, in studies of rats exhibiting insulin resistance (IR), there is an improvement in peripheral sensitivity to this hormone after treatment with low frequency electroacupuncture (EA) when administered three times a week for four or five weeks, ${ }^{39}$ and it returns to normal with five treatments per week. ${ }^{40}$ It should be noted that a doseresponse relationship is observed between the number of treatments with EA and the improvement in insulin sensitivity. ${ }^{39}$ It has also been shown that low frequency EA has improved insulin sensitivity in mice with Diabetes mellitus type 2. This effect is mediated by the Sirtuin-1 regulation of skeletal muscle and the Peroxisome proliferatoractivated receptor-gamma coactivator 1 alpha. ${ }^{41}$

In TCM, the term "acupuncture points" is used, referring to the position of the needle in certain areas of somatic innervation in different areas of the body, such as abdominal muscles and legs. There are points that correspond to the sympathetic innervation of the ovary, so they exert an effect on its function by modulating efferent sympathetic activity. ${ }^{42-44}$ Studies in rats have shown an increase in ovarian blood flow with the use of low frequency EA. ${ }^{42,43}$

Acupuncture can, in turn, modulate endocrine and metabolic functions through mechanisms that involve the Central Nervous 
System (CNS). ${ }^{32}$ For example, acupuncture induces the release of neuropeptides from the CNS that produce functional changes in organ systems. $^{45}$

One of the most interesting theories about the mechanisms of action of acupuncture in the body is related to the opioid system (OS). The OS performs actions on behaviour, regulation of appetite, body temperature, respiratory activity, regulating mechanisms of the sleep-wake cycles, mood and cognition. ${ }^{46-49}$ Moreover, the OS plays a role in carbohydrate metabolism, insulin resistance and follicular maturation of the reproductive cycle. ${ }^{50}$

The opioid system is composed of several types of receptors that include: $\mathrm{Mu}(\mu)$, kappa $(\kappa)$, and delta $(\delta)$ and opioid receptor like1 (ORL1). ${ }^{50}$ The endogenous ligands for these receptors include peptides of various families; one of them is the group of endorphins. ${ }^{50}$ The $\beta$-Endorphin is the main molecule of this family.

Polycystic Ovarian Syndrome (PCOS) is one of the most frequent disorders in women of reproductive age who also suffer from infertility. ${ }^{51}$ It is accompanied by alterations and hormonal imbalances such as hyperinsulinemia, hyperandrogenism and alterations in the secretion of gonadotropins..$^{51,52}$ In a review by Eyvazzadeh et al., ${ }^{50}$ they emphasise that the OS has a role in the pathogenesis of PCOS:

a) The opioid activity seems to be altered in women with PCOS, both central as peripheral; $53-55$

b) Opioids may have an impact on the abnormal secretion pattern of gonadotropins and the metabolic imbalance observed in women with PCOS; ${ }^{56,57}$

c) Opioid synthesizing cells of the pituitary gland have insulin receptors; ${ }^{58}$

d) The $\beta$-endorphin has inhibitory effects on the release of gonadotropin-releasing hormone $(\mathrm{GnRH})$ and stimulates the release of insulin and glucagon. ${ }^{59}$

The hypothalamic $\beta$-endorphin interacts with the hypothalamicpituitary-ovary axis by exerting a tonic inhibitory effect on the pulsatile secretion of GnRH and, in turn, on the release of LH by the pituitary gland ${ }^{60}$ Opioid receptor antagonists have been shown to regulate the menstrual cycle, induce ovulation, and lower levels of testosterone, insulin, and the LH/FSH ratio. ${ }^{61,62}$ Acupuncture can modulate the central production of $\beta$-endorphin and its secretion; therefore, they have an effect on the release of GnRH by the hypothalamus and gonadotropins by the pituitary gland ${ }^{32}$ this has been demonstrated by decreasing the LH/FSH ratio after low frequency EA. ${ }^{63}$

It is believed that the circulating $\beta$-endorphin is involved in hyperinsulinemia in patients with PCOS. Low-frequency AE decreases circulating levels of $\beta$-endorphin in women with PCOS and may decrease hyperinsulinemia and increase insulin clearance or insulin sensitivity. ${ }^{63}$

The combination of exercise and low frequency $\mathrm{AE}$ has been shown to increase the hypothalamic secretion of $\beta$-Endorphin and the decrease in blood pressure and sympathetic nervous activity. ${ }^{32}$ Frequent sessions of low frequency EA and physical exercise significantly decrease the activity of sympathetic nerves when measured by microneurography in women with PCOS. ${ }^{64}$ This may explain, in part, the decrease in serum testosterone levels and the regulation of the menstrual cycle following low frequency EA in conjunction with exercise for patients with PCOS. ${ }^{65}$

\section{Acupuncture and infertility: applying current results}

Acupuncture has different applications and although Western scientific bases are known only in some of them, its use is increasingly accepted in different medical specialties.

Infertility is a multifactorial condition and it is quite often not possible to correct all the causes so therefore people turn to techniques or procedures that eliminate the causal factor, as is the case of ART. Acupuncture, on the other hand, has been seen to improve or favourably regulate the hypothalamic-pituitary-gonadal axis (HPG axis) thus helping the reproductive function.

If we take the cases of infertility of anovulatory origin due to PCOS or in patients with ovarian dysfunction as a basis, it has been observed that repeated treatments with acupuncture decrease the serum levels of total testosterone and other sex steroids, also reduce the proportion of LH/FSH and regulates the menstrual cycle without adverse side effects. ${ }^{63,66,67}$ This can also be reflected in an improvement in fertility by correcting the menstrual cycles of the patients.

In a controlled and randomized study by Jedel et al., ${ }^{65}$ eighty-four women diagnosed with PCOS were divided into three groups: thirtythree patients received treatment with low-frequency EA, thirty-four performed physical exercise and seventeen women without any form of intervention. It showed that EA was superior to physical exercise, improved hyperandrogenism and regulated the menstrual cycle more effectively than non-intervention. ${ }^{65}$

Johansson et al. ${ }^{68}$ conducted a randomized controlled trial where they observed that repeated treatments with acupuncture improve ovulation in thin and overweight women with PCOS, and there is a decrease in serum estrone, estrone sulphate, oestradiol and most of the androgen levels measured.

Jiang et al.$^{69}$ conducted a study on the treatment of infertility in patients with PCOS. They included 120 randomized women in 3 groups (clomiphene group, acupuncture-moxibustion group + Chinese Medicine, and clomiphene + acupuncture-moxibustion + Chinese Medicine group), which showed that the combination of acupuncture, Chinese Medicine and clomiphene improves pregnancy rates and reduces early abortion rates by improving cervical mucus, thickness and endometrial morphology.

On the other hand, a recent Cochrane meta-analysis that includes 5 randomized controlled trials with 413 women, concluded that current evidence is of very low quality and that there is not a sufficient number of studies to support the use of acupuncture for the treatment of Ovulation disorders in women with PCOS. ${ }^{70}$

A couple of pilot studies are underway that have adequate design and methodology on treatment with acupuncture for women with PCOS, where one of them focuses on the management of infertility. ${ }^{71,72}$ However, until the results have been published, we cannot make conclusions or make decisions about acupuncture in PCOS.

However, the use of acupuncture is not limited to regulating the menstrual cycle and treating ovulation disorders. Its application together with ART is justified by several points: ${ }^{12}$

i. It reduces patient stress during and around the time of embryo transfer, possibly improving pregnancy rates $;^{73}$

ii. Acupuncture improves uterine flow and reduces the impedance of the uterine artery, possibly aiding implantation $;^{74}$ 
iii. Improves the hormonal balance in the stage of embryo implantation; ${ }^{12}$

iv. Promotes the release of neurotransmitters mediating hormonal release: ${ }^{75}$

v. It is possible to regulate the levels of stem cell factor in follicular fluid and serum, improving oocyte quality. ${ }^{17}$

In a systematic review and meta-analysis by Shen et al. ${ }^{12}$ where 21 controlled and randomized studies involving a total of 5,428 participants are included, it was found that when acupuncture is performed only around embryo transfer there is no improvement in clinical pregnancy rates when compared with controls without treatment (RR 1.24, 95\% CI 1.02-1.50, I2 = 64\%, 10 studies, 2,226 participants). Nor did they observe statistical differences in the subgroups of non-blinded studies (RR 1.54, IC95\% 1.26-1.88, I2 = $0 \%, 5$ studies, 757 participants), of blind studies for physicians (RR 1.04 , IC95\% 0.82-1.33, I2 $=67 \%, 5$ studies, 1,469 participants) or double blind (RR 1.05, 95\% CI 0.89-1.25, I2 = 0\%, 1 study, 599 participants). However, a statistically significant difference is observed when acupuncture is administered 30 minutes after the embryo transfer at the same time as in the implantation phase (RR 1.76 , IC95\% $1.22-2.55$, I2 $=14 \%, 2$ studies, 417 participants) and when used from the follicular phase and around the embryo transfer (RR 1.56, 95\% CI 1.04-2.33, I2 = 50\%, 2 studies, 284 participants). ${ }^{12}$ The authors highlight the positive effects of acupuncture when used from the follicular phase to the embryo transfer of IVF procedures and that its potential benefits on the hypothalamic-pituitary-gonadal axis are still unknown in their entirety. ${ }^{12}$

In a retrospective cohort study evaluating the effects of TCM on the results of IVF procedures, ${ }^{76} 1,231$ patients are included divided into cycles with their own oocytes $(\mathrm{n}=1069)$ and cycles of donated oocytes $(n=162)$. In the cycles where there was no donation, 580 patients participated as a control group, 370 were given an acupuncture treatment (ACU) and 119 received Chinese herbal treatment in addition to acupuncture (WSTCM). The live birth rate was significantly higher in the WSTCM group $(61.3 \%)$ when compared to the control group (48.2\%) or the ACU group (50.8\%), $\mathrm{p}=0.03$. In addition, a decrease in spontaneous abortion rate was observed $(\mathrm{p}=0.02)$ in this same group (WSTCM). Of the donation cycles, 104 recipients were included as a control group 37 in the ACU group and 21 in the WSTCM group. It is worth noting that the odds of live birth were associated with the WSTCM group when compared with the other two (OR 3.72, 95\% CI 1.05-13.24). However, the small sample size limits these observations.

\section{Acupuncture and infertility: problematic}

There are many deficiencies in current acupuncture studies. For example, some authors have shown that the efficacy of acupuncture varies proportionally to the dose used. ${ }^{77,78}$ In other words, the higher the dose, the better the results. The STRICTA guidelines (Standards for Reporting Interventions in Clinical Trials of Acupuncture ${ }^{79}$ were designed to improve the quality and transparency of interventions and controlled studies of acupuncture, so that they are interpreted and replicated with accuracy.

The STRICTA guidelines provide authors with a way to structure the reports of acupuncture interventions through a set with a minimum number of items. ${ }^{79}$ This not only facilitates transparency, but also describes all the steps necessary to replicate the studies.
Recently, the STRICTA group has worked in collaboration with the CONSORT group (Consolidated Standards of Reporting Trials), the Chinese Cochrane Centre and the Chinese EvidenceBased Medicine Centre, which has led to the development of new guidelines with explanations and examples for a good clinical report. ${ }^{79}$ STRICTA's revised guidelines include 6 items and 17 sub-items that describe the acupuncture techniques, descriptions of the needles used, the treatment regimen, the experience of the acupuncturist and the control or comparison interventions. ${ }^{79}$

An example of the lack or urgent need to implement these guidelines is shown by Wu et al..$^{30}$ Her group reviewed 31 randomized controlled studies that included 2371 women with PCOS. Of the 31 studies, three were conducted in Sweden, $, 4,65,80$ one in the USA, ${ }^{81}$ one in Iran ${ }^{82}$ and the rest in China. Only six of the studies followed the STRICTA guidelines. ${ }^{64,65,80-83}$

Future studies should aim to complete the minimum requirements to provide high-quality work. It is necessary to include all the information related to the treatment and to monitor for an optimal period that allows us to evaluate the effectiveness of acupuncture in patients with infertility.

\section{Acupuncture and infertility: how to demonstrate or rule out its effect.}

As described in previous paragraphs, the bases to justify the use of acupuncture in conjunction with the ART are diverse: from the improvement in the uterine flow and the regulation of the HPO axis, to the improvement of the oocyte quality and the decrease of patient stress. However, the lack of consistent and correct methodology for the design of study protocols in acupuncture is the basis from which new trials should start to be developed.

In order to organize and justify the positive effect of acupuncture on ART, we need to consider the importance of evaluating and describing changes in the following aspects that can offer us a better perspective on their role for the improvement of results. They are listed as follows:

i. Changes related to the TRA cycle
a. Effects on controlled ovarian hyperstimulation
b. Effects on oocyte quality
c. Effects on embryo quality

ii. Changes related to the uterine vasculature
a. Effect on the resistance of the uterine arteries
b. Effect on endometrial flow
c. Effect on vascular growth factors

iii. Changes related to stress
a. Effect on biochemical markers of stress
b. Psychological effect

iv. Final results of the TRA cycle
a. Pregnancy rate
b. Implantation rate 


\section{c. Abortion rate \\ d. Clinical pregnancy rate}

As described on the known mechanism of action of acupuncture and on the improvement in physiological and psychological aspects, we believe that in this way we can obtain an overview that would give grounds for discarding or supporting its use in ART.

Finally, it is important to highlight another negative aspect in current studies of acupuncture and which will be a fundamental point in the design of future research projects: the "one size fits all" protocols (uni-size). This refers to the use of a single acupuncture treatment protocol for all patients regardless of age, weight or diagnosis.

In the same way as in Western Medicine an etiological diagnosis is made to treat the cause in the vast majority of cases, Chinese Medicine also makes a diagnosis that in many cases can be: Kidney, Spleen deficiency, Qi stagnation of liver, blood stasis or, a combination of these ${ }^{84}$ For this reason, Brenner ${ }^{85}$ pointed out that this behaviour of "one size fits all approach" only gives us information on the protocol applied in a given study and not of the potential that acupuncture may have as an as an adjuvant. We emphasize, therefore, the need to adapt treatment in order to obtain the best results.

In addition, although the best results have been demonstrated when acupuncture is used from the follicular phase to the embryo transfer, ${ }^{12}$ the recommendation, once the diagnosis is made, is to apply the treatment for a period of up to 3 months for better results. ${ }^{86}$

Finally, by applying the STRICTA guidelines correctly and, above all, giving a detailed justification and description, it will be possible to observe the presence or not of a positive effect of TCM in its acupuncture modality, on the results of ART.

\section{Conclusion}

Despite great scientific and technological advances, assisted reproduction procedures continue to show relatively low results when comparing the cost-benefit in terms of live birth at home.

The use of acupuncture as a coadjuvant treatment in assisted reproduction procedures has been suggested as a low-cost strategy that can improve results. However, the lack of adequate design and methodology in current studies, as well as not adapting acupuncture treatments on a per case basis, result in a lack of consistency and reproducibility of the results.

\section{Acknowledgments}

None.

\section{Conflicts of interest}

The authors declare that they have no conflict of interest.

\section{References}

1. Inhorn MC, Patrizio P. Infertility around the globe: new thinking on gender, reproductive technologies and global movements in the 21st century. Hum Reprod Update. 2015;21(4):411-426.

2. Ombelet W, Cooke I, Dyer S, et al. Infertility and the provision of infertility medical services in developing countries. Hum Reprod Update. 2008;14(6):605-621.

3. Nachtigall RD. International disparities in access to infertility services. Fertility and sterility. 2006;85(4):871-875.
4. Mascarenhas MN, Flaxman SR, Boerma T, et al. National, regional, and global trends in infertility prevalence since 1990: a systematic analysis of 277 health surveys. PLoS Med. 2012;9(12):e1001356.

5. Societies iFoF. IFFS Surveillance 2016. Global Reproductive Health. 2016;1(1):1-143.

6. Kazemijaliseh H, Ramezani Tehrani F, Behboudi-Gandevani S, et al. The Prevalence and Causes of Primary Infertility in Iran: A PopulationBased Study. Glob J Health Sci. 2015;7(6):226-232.

7. Ombelet W. Global access to infertility care in developing countries: a case of human rights, equity and social justice. Facts Views Vis Obgyn. 2011;3(4):257-266.

8. Naab F, Brown R, Heidrich S. Psychosocial health of infertile Ghanaian women and their infertility beliefs. J Nurs Scholarsh. 2013;45(2):132140 .

9. Gannon K, Glover L, Abel P. Masculinity, infertility, stigma and media reports. Soc Sci Med. 2004;59(6):1169-1175.

10. Szamatowicz M. Assisted reproductive technology in reproductive medicine - possibilities and limitations. Ginekol Pol. 2016;87(12):820 823.

11. Calhaz-Jorge C, de Geyter C, Kupka MS, de Mouzon J, et al. Assisted reproductive technology in Europe, 2012: results generated from European registers by ESHRE. Hum Reprod. 2016;31(8):1638-1652.

12. Shen $\mathrm{C}, \mathrm{Wu} \mathrm{M}$, Shu $\mathrm{D}$, et al. The role of acupuncture in in vitro fertilization: a systematic review and meta-analysis. Gynecol Obstet Invest. 2015;79(1):1-12.

13. Adams J, Lui CW, Sibbritt D, et al. Women's use of complementary and alternative medicine during pregnancy: a critical review of the literature. Birth. 2009;36(3):237-245.

14. Smith CA, Bateson DJ, Weisberg E. A survey describing the use of complementary therapies and medicines by women attending a family planning clinic. BMC Complement Altern Med. 2013;13:224.

15. Gollschewski S, Anderson D, Skerman H, et al. Associations between the use of complementary and alternative medications and demographic, health and lifestyle factors in mid-life Australian women. Climacteric. 2005;8(3):271-278.

16. $\mathrm{Ng} \mathrm{EH}$, So WS, Gao J, et al. The role of acupuncture in the management of subfertility. Fertil Steril. 2008;90(1):1-13.

17. Chen J, Liu LL, Cui W, et al. Effects of electroacupuncture on in vitro fertilization-embryo transfer (IVF-ET) of patients with poor ovarian response. Zhongguo Zhen Jiu. 2009;29(10):775-779.

18. El-Toukhy T, Sunkara SK, Khairy M, et al. A systematic review and meta-analysis of acupuncture in in vitro fertilisation. BJOG. 2008;115(10):1203-1213.

19. Manheimer E, van der Windt D, Cheng K, et al. The effects of acupuncture on rates of clinical pregnancy among women undergoing in vitro fertilization: a systematic review and meta-analysis. Hum Reprod Update. 2013;19(6):696-713.

20. Zheng $\mathrm{CH}$, Zhang MM, Huang GY, et al. The role of acupuncture in assisted reproductive technology. Evid Based Complement Alternat Med. 2012;2012:543924.

21. El-Toukhy T, Khalaf Y. The impact of acupuncture on assisted reproductive technology outcome. Curr Opin Obstet Gynecol. 2009;21(3):240-246.

22. Messinis IE, Messini CI, Daponte A, et al. The current situation of infertility services provision in Europe. Eur J Obstet Gynecol Reprod Biol. 2016. 
23. Vahratian A, Smith YR, Dorman M, et al. Longitudinal depressive symptoms and state anxiety among women using assisted reproductive technology. Fertil Steril. 2011;95(3):1192-1194.

24. Coughlan C, Walters S, Ledger W, et al. A comparison of psychologica stress among women with and without reproductive failure. Int $J$ Gynaecol Obstet. 2014;124(2):143-147.

25. Steptoe PC, Edwards RG. Birth after the reimplantation of a human embryo. Lancet. 1978;2(8085):366.

26. Jones HW, Cooke I, Kempers R, Brinsden PS, D. Internationa Federation of Fertility Societies Surveillance 2010: preface. Fertil Steril. 2011;95(2):491.

27. Papaleo E, Pagliardini L, Vanni VS, et al. A direct healthcare cost analysis of the cryopreserved versus fresh transfer policy at the blastocyst stage. Reprod Biomed Online. 2017;34(1):19-26

28. Edwards RG. Clinical approaches to increasing uterine receptivity during human implantation. Hum Reprod. 1995;10 Suppl 2:60-66.

29. Fertilidad SEd. Registro Nacional de Actividad 2014 - Registro SEF Sociedad Española de Fertilidad; 2016.

30. Wu Y, Robinson N, Hardiman PJ, et al. Acupuncture for treating polycystic ovary syndrome: guidance for future randomized controlled trials. J Zhejiang Univ Sci B. 2016;17(3):169-180.

31. Stener-Victorin E, Jedel E, Manneras L. Acupuncture in polycystic ovary syndrome: current experimental and clinical evidence. $J$ Neuroendocrinol. 2008;20(3):290-298.

32. Raja-Khan N, Stener-Victorin E, Wu X, et al. The physiological basis of complementary and alternative medicines for polycystic ovary syndrome. Am J Physiol Endocrinol Metab. 2011;301(1):E1-E10.

33. White A, Cummings M, Barlas P, et al. Defining an adequate dose of acupuncture using a neurophysiological approach--a narrative review of the literature. Acupunct Med. 2008;26(2):111-120.

34. Sherman KJ, Cherkin DC, Ichikawa L, et al. Treatment expectations and preferences as predictors of outcome of acupuncture for chronic back pain. Spine (Phila Pa 1976). 2010;35(15):1471-1477.

35. Kagitani F, Uchida S, Hotta $\mathrm{H}$, et al. Manual acupuncture needle stimulation of the rat hindlimb activates groups I, II, III and IV single afferent nerve fibers in the dorsal spinal roots. Jpn J Physiol. 2005;55(3):149-155.

36. Sato A, Sato Y. Regulation of regional cerebral blood flow by cholinergic fibers originating in the basal forebrain. Neurosci Res. 1992;14(4):242274 .

37. Jansen G, Lundeberg T, Kjartansson J, et al. Acupuncture and sensory neuropeptides increase cutaneous blood flow in rats. Neurosci Lett. 1989;97(3):305-309.

38. Holmang A, Mimura K, Lonnroth P. Involuntary leg movements affect interstitial nutrient gradients and blood flow in rat skeletal muscle. $J$ Appl Physiol (1985). 2002;92(3):982-988.

39. Manneras L, Jonsdottir IH, Holmang A, et al. Low-frequency electro-acupuncture and physical exercise improve metabolic disturbances and modulate gene expression in adipose tissue in rats with dihydrotestosterone-induced polycystic ovary syndrome. Endocrinology. 2008;149(7):3559-3568.

40. Johansson J, Feng Y, Shao R, et al. Intense electroacupuncture normalizes insulin sensitivity, increases muscle GLUT4 content, and improves lipid profile in a rat model of polycystic ovary syndrome. $\mathrm{Am}$ J Physiol Endocrinol Metab. 2010;299(4):E551-E559.
41. Liang F, Chen R, Nakagawa A, et al. Low-Frequency Electroacupuncture Improves Insulin Sensitivity in Obese Diabetic Mice through Activation of SIRT1/PGC-1alpha in Skeletal Muscle. Evid Based Complement Alternat Med. 2011;2011:735297.

42. Stener-Victorin E, Fujisawa S, Kurosawa M. Ovarian blood flow responses to electroacupuncture stimulation depend on estrous cycle and on site and frequency of stimulation in anesthetized rats. $J$ Appl Physiol (1985). 2006;101(1):84-91.

43. Stener-Victorin E, Kobayashi R, Kurosawa M. Ovarian blood flow responses to electro-acupuncture stimulation at different frequencies and intensities in anaesthetized rats. Auton Neurosci. 2003;108(1-2):50-56.

44. Stener-Victorin E, Lundeberg T, Waldenstrom U, et al. Effects of electro-acupuncture on nerve growth factor and ovarian morphology in rats with experimentally induced polycystic ovaries. Biol Reprod. 2000;63(5):1497-1503.

45. Han JS. Acupuncture: neuropeptide release produced by electrical stimulation of different frequencies. Trends Neurosci. 2003;26(1):1722 .

46. Fadda P, Tortorella A, Fratta W. Sleep deprivation decreases mu and delta opioid receptor binding in the rat limbic system. Neurosci Lett. 1991;129(2):315-317.

47. Guillemin R, Vargo $\mathrm{T}$, Rossier $\mathrm{J}$, et al. Beta-Endorphin and adrenocorticotropin are selected concomitantly by the pituitary gland. Science. 1977;197(4311):1367-1379.

48. Panksepp J. Neuroscience. Feeling the pain of social loss. Science. 2003;302(5643):237-239

49. Kennedy SE, Koeppe RA, Young EA, et al. Dysregulation of endogenous opioid emotion regulation circuitry in major depression in women. Arch Gen Psychiatry. 2006;63(11):1199-1208.

50. Eyvazzadeh AD, Pennington KP, Pop-Busui R, et al. The role of the endogenous opioid system in polycystic ovary syndrome. Fertility and sterility. 2009;92(1):1-12.

51. Carmona-Ruiz IO, Saucedo-de la Llata E, Moraga-Sanchez MR, et al. Polycystic ovary syndrome: is there a rise in the prevalence?. Ginecol Obstet Mex. 2015;83(12):750-759.

52. Saucedo de la Llata E, Moraga-Sanchez MR, Romeu-Sarrio A, et al LH-FSH ratio and Polycystic Ovary Syndrome: A forgotten test? Ginecol Obstet Mex. 2016;84(2):84-94.

53. Lanzone A, Fulghesu AM, Fortini A, et al. Effect of opiate receptor blockade on the insulin response to oral glucose load in polycystic ovarian disease. Human reproduction. 1991;6(8):1043-1049.

54. Fulghesu AM, Lanzone A, Cucinelli F, et al. Long-term naltrexone treatment reduces the exaggerated insulin secretion in patients with polycystic ovary disease. Obstet Gynecol. 1993;82(2):191-197.

55. Lanzone A, Apa R, Fulghesu AM, et al. Long-term naltrexone treatment normalizes the pituitary response to gonadotropin-releasing hormone in polycystic ovarian syndrome. Fertility and sterility. 1993;59(4):734 737.

56. Cumming DC, Reid RL, Quigley ME, et al. Evidence for decreased endogenous dopamine and opioid inhibitory influences on LH secretion in polycystic ovary syndrome. Clinical endocrinology. 1984;20(6):643648.

57. Guido M, Romualdi D, Lanzone A. Role of opioid antagonists in the treatment of women with glucoregulation abnormalities. Curr Pharm Des. 2006;12(8):1001-1012. 
58. Lackey BR, Gray SL, Henricks DM. The insulin-like growth factor (IGF) system and gonadotropin regulation: actions and interactions. Cytokine Growth Factor Rev. 1999;10(3-4):201-217.

59. Snowden EU, Khan-Dawood FS, Dawood MY. The effect of naloxone on endogenous opioid regulation of pituitary gonadotropins and prolactin during the menstrual cycle. The Journal of clinical endocrinology and metabolism. 1984;59(2):298-302.

60. Jenkins PJ, Grossman A. The control of the gonadotrophin releasing hormone pulse generator in relation to opioid and nutritional cues. Human reproduction. 1993;8 Suppl 2:154-161.

61. Ahmed MI, Duleba AJ, El Shahat O, et al. Naltrexone treatment in clomiphene resistant women with polycystic ovary syndrome. Human reproduction. 2008;23(11):2564-2569.

62. Fruzzetti F, Bersi C, Parrini D, et al. Effect of long-term naltrexone treatment on endocrine profile, clinical features, and insulin sensitivity in obese women with polycystic ovary syndrome. Fertility and sterility. 2002;77(5):936-944.

63. Stener-Victorin E, Waldenstrom U, Tagnfors U, et al. Effects of electroacupuncture on anovulation in women with polycystic ovary syndrome. Acta obstetricia et gynecologica Scandinavica. 2000;79(3):180-188.

64. Stener-Victorin E, Jedel E, Janson PO, et al. Low-frequency electroacupuncture and physical exercise decrease high muscle sympathetic nerve activity in polycystic ovary syndrome. Am J Physiol Regul Integr Comp Physiol. 2009;297(2):R387-R395.

65. Jedel E, Labrie F, Oden A, et al. Impact of electro-acupuncture and physical exercise on hyperandrogenism and oligo/amenorrhea in women with polycystic ovary syndrome: a randomized controlled trial. Am J Physiol Endocrinol Metab. 2011;300(1):E37-E45.

66. Chen BY, Yu J. Relationship between blood radioimmunoreactive betaendorphin and hand skin temperature during the electro-acupuncture induction of ovulation. Acupunct Electrother Res. 1991;16(1-2):1-5.

67. Mo X, Li D, Pu Y, et al. Clinical studies on the mechanism for acupuncture stimulation of ovulation. J Tradit Chin Med. 1993;13(2):115-119.

68. Johansson J, Redman L, Veldhuis PP, et al. Acupuncture for ovulation induction in polycystic ovary syndrome: a randomized controlled trial. Am J Physiol Endocrinol Metab. 2013;304(9):E934-E943.

69. Jiang D, Zhang Y, Wu X, et al. Infertility in polycystic ovary syndrome treated with acupuncture and clomiphene: a randomized controlled trial. Zhongguo Zhen Jiu. 2015;35(2):114-118.

70. $\mathrm{Lim} \mathrm{CE}, \mathrm{Ng} \mathrm{RW}, \mathrm{Xu} \mathrm{K}$, et al. Acupuncture for polycystic ovarian syndrome. Cochrane Database Syst Rev. 2016(5):CD007689.

71. Zheng Y, Stener-Victorin E, Ng EH, et al. How does acupuncture affect insulin sensitivity in women with polycystic ovary syndrome and insulin resistance? Study protocol of a prospective pilot study. BMJ Open. 2015;5(4):e007757.

72. Kuang H, Li Y, Wu X, et al. Acupuncture and clomiphene citrate for live birth in polycystic ovary syndrome: study design of a randomized controlled trial. Evid Based Complement Alternat Med. 2013;2013:527303.

73. Balk J, Catov J, Horn B, et al. The relationship between perceived stress, acupuncture, and pregnancy rates among IVF patients: a pilot study. Complement Ther Clin Pract. 2010;16(3):154-157.

74. Cakmak YO. Electroacupuncture reduces uterine artery blood flow impedance. Taiwan J Obstet Gynecol. 2010;49(3):390.

75. Guo J, Li D, Zhang QF. Acupuncture intervention combined with assisted reproductive technology: its different effects at different time points during the in vitro fertilization-embryo transfer course. Zhong Xi Yi Jie He Xиe Bao. 2008;6(12):1211-1216.

76. Hullender Rubin LE, Opsahl MS, Wiemer KE, et al. Impact of whole systems traditional Chinese medicine on in-vitro fertilization outcomes. Reprod Biomed Online. 2015;30(6):602-612.

77. Humaidan P, Brock K, Bungum L, et al. Pain relief during oocyte retrieval--exploring the role of different frequencies of electroacupuncture. Reprod Biomed Online. 2006;13(1):120-125.

78. Anderson BJ, Rosenthal L. Acupuncture and IVF Controversies. Fertil Steril. 2007;87(4):1000.

79. MacPherson H, Altman DG, Hammerschlag R, et al. Revised STandards for Reporting Interventions in Clinical Trials of Acupuncture (STRICTA): extending the CONSORT statement. PLoS Med. 2010;7(6):e1000261.

80. Johansson J, Stener-Victorin E. Polycystic ovary syndrome: effect and mechanisms of acupuncture for ovulation induction. Evid Based Complement Alternat Med. 2013;2013:762615.

81. Pastore LM, Williams CD, Jenkins J, et al. True and sham acupuncture produced similar frequency of ovulation and improved LH to FSH ratios in women with polycystic ovary syndrome. The Journal of clinical endocrinology and metabolism. 2011;96(10):3143-3150.

82. Rashidi BH, Tehrani ES, Hamedani NA, et al. Effects of acupuncture on the outcome of in vitro fertilisation and intracytoplasmic sperm injection in women with polycystic ovarian syndrome. Acupunct Med. 2013;31(2):151-156.

83. Zheng $\mathrm{YH}$, Wang $\mathrm{XH}$, Lai $\mathrm{MH}$, et al. Effectiveness of abdominal acupuncture for patients with obesity-type polycystic ovary syndrome: a randomized controlled trial. J Altern Complement Med. 2013;19(9):740 745.

84. Zheng $\mathrm{CH}$, Zhang J, Wu J, et al. The effect of transcutaneous electrical acupoint stimulation on pregnancy rates in women undergoing in vitro fertilization: a study protocol for a randomized controlled trial. Trials. 2014; $15: 162$.

85. Brenner Z. Acupuncture and IVF trial. Fertil Steril. 2009;92(2):e27.

86. yuan CZyx. Zhong yi fu ke xue: Sichuan ren min chu ban she. 1981. 206 p. 